\title{
Virtual Reality Genres
}

\section{Comparing Preferences in Immersive Experiences and Games}

\author{
Maxwell Foxman \\ School of Journalism and \\ Communication \\ University of Oregon \\ Eugene Oregon USA \\ mfoxman@uoregon.edu \\ Brian Klebig \\ Communication \\ Bethany Lutheran College \\ Mankato Minnesota USA \\ bklebig@blc.edu
}

\author{
Alex P. Leith \\ Media Studies \\ Southern Illinois University \\ Edwardsville Illinois USA \\ aleith@siue.edu \\ Vivian Hsueh Hua Chen \\ Wee Kim Wee School of \\ Communication and Information \\ Nanyang Technological \\ University \\ Jurong West Singapore \\ chenhh@ntu.edu.sg
}

\author{
David Beyea \\ Communication \\ University of Wisconsin \\ Whitewater Wisconsin USA \\ beyead@uww.edu \\ Rabindra Ratan \\ Media \& Information \\ Michigan State University \\ East Lansing Michigan USA \\ rar@msu.edu
}

\begin{abstract}
Even though virtual reality (VR) shares features with video games, it offers a wider range of experiences. There is currently no cohesive classification for commercial VR offerings. As a first step to account for this deficiency, the work in progress considers the relationship between game genres and users' ratings and downloads of VR experiences. We found Action, Shooter, and Simulation to be the most frequently downloaded genres; Action and Music/Rhythm the most highly rated; and Simulation and Music/Rhythm to occur at a statistically higher rate in VR compared to non-VR. Finally, we learned that VR experiences are less likely to receive positive ratings than $2 \mathrm{D}$ games. The findings can inform developers' marketing decisions based on demand.
\end{abstract}

\section{CCS CONCEPTS}

- Human-centered computing $\rightarrow$ Human computer interaction (HCI); Interaction paradigms; Virtual Reality;

\section{KEYWORDS}

Virtual Reality; Game Genres; Video Games; Steam

\section{ACM Reference format:}

Maxwell Foxman, Alex P. Leith, David Beyea, Brian Klebig, Vivian Hsueh Hua Chen, and Rabindra Ratan. 2020. Virtual Reality Genres: Comparing Preferences in Immersive Experiences and Games. In Extended Abstracts of the 2020 Annual Symposium on Computer-Human Interaction in Play (CHI PLAY '20) November 2-4, 2020, Virtual Event, Canada. ACM, New York, NY, USA, 5 pages. https://doi.org/10.1145/3383668.3419881

Permission to make digital or hard copies of part or all of this work for personal or classroom use is granted without fee provided that copies are not made or distributed for profit or commercial advantage and that copies bear this notice and the full citation on the first page. Copyrights for third-party components of this work must be honored. For all other uses, contact the Owner/Author.

CHI PLAY' '20 EA, November 2-4, 2020, Virtual Event, Canada

(c) 2020 Copyright is held by the owner/author(s).

ACM ISBN 978-1-4503-7587-0/20/11.

https://doi.org/10.1145/3383668.3419881

\section{Introduction}

While virtual reality (VR) is inextricably linked to video games, it seems to defy traditional game genres by offering new types of engagement, control, and transport that change user experience. Our work in progress begins to address this by using Steam and SteamSpy API data to analyze and compare users' ratings and downloads across genres of VR and non-VR games. The findings can inform developers' marketing decisions based on demand, allow for more intelligently created and stylized titles, and identify merchandising opportunities. The study also provides publishers information about attractive and appropriate genre labels (or tags) when they release content. Future research will update and define popular VR genres, and build new frameworks to systematically apprehend user experience and products.

\section{Related Works}

While genres are important in defining games, relating them to VR is complicated by a lack of standards and focus on distinct affordances over content.

\subsection{Game Genres}

Genre, or the "style" or "type" [12] of a game, is an essential classification system for the medium. Genre choice is linked to player personality/preference [13,22], predicts addiction [6], and enhances creative and cognitive abilities [5]. Similarly, "[v]ideo game genres provide a design lens through which developers can analyze gameplay preferences and player experiences" [17:2265].

Despite this, there is no single rubric for game genres due to insufficient industry criteria for identifying titles. Many games do not conform to traditional genre typologies [30:773], and are instead compared to familiar predecessors: for instance, "Roguelike" references similar patterns from the classic game Rogue. It is particularly difficult to separate genres from gameplay: 
a game may be classified as "Action/Adventure" if the player advances an avatar gradually through levels to confront a final villain; but that same content will be defined as a "Shooter" if the player takes a first-person perspective. Ye [30] suggests this confusion stems from the tension between traditional genre conventions, interactive metaphors, game pace, and controllers, all of which influence player experience, but are "not clearly or consistently defined" [12:176].

Accordingly, numerous genre classification frameworks of varying lengths exist $[7,18,29]$, or genre tends to be integrated into broader design strategies to identify player preferences and experience (PX) [26,27], such as the Game Elements-Attribute Model [12]. The profusion of models underscores the need for clarity among styles, features, and experiences of games generally.

\subsection{VR Elements and Genre}

This is magnified in VR because it shares fundamental characteristics with video game play including narrativity, simulation, interactivity, and intelligence [24]. The diffusion of commercial headsets also occurred in a platformized environment which is "contingent" $[8,20]$ upon multi-sided markets like Steam's digital game store for distribution. Consequently, VR content is linked to "how game mechanics work" [9:367] even though it may serve different ends, as is the case with social VR [14].

However, studies of VR design center on unique affordances of room-scale head-mounted displays (HMDs) [1,4]. Analyses emphasize attributes, such as presence [11], immersion [3], embodiment [2,16], transportation [10] and perspective-taking [15] over specific styles. Comparative work between games and VR focus on characteristics like levels of immersion, flow [21,25] and presence [23].

Thus, there is a gap between how genres are defined in VR experiences and their effects on users. In this nascent industry, developers struggle to situate their work within already vague game categories. Further, companies cannot deduce what styles and content are explicitly popular to VR. Recognizing the inconsistencies of genre classification systems, we aim to build a more inclusive framework that encompasses affordances, mechanics, and key features of the medium for assessing VR content.

Because game genres are a familiar and widely used means for developers to market and classify VR titles, our analysis starts with one existing game genre classification to ask:

$R Q_{1}$ : What traditional game genres are most common in Steamprovided VR experiences?

$R Q_{2}$ : Are user ratings and downloads higher for certain traditional genres in VR experiences?

$R Q_{3}$ : Do VR and non-VR experiences differ with respect to traditional game genre availability and user ratings?

\section{Methods}

Seeking answers, we analyzed content from the Steam game market.

\subsection{Data Collection}

Steam has become the central hub for PC games, especially VR, and houses the largest library compatible with a range of accessible HMDs. Using the Steam API, users are able to get a list of all games hosted on Steam.

We limited our query to the most popular experiences within Steam's "VR Only" category. In order to facilitate clear comparisons, we excluded games with both VR and non-VR support, and will add this category in future work. We pulled ownership (download) estimates from SteamSpy's third-party API. The popularity threshold for VR experiences was 50,000100,000 estimated ownership $(N=141$; approximately $3.25 \%$ of all VR Only experiences). The threshold for all games was 500,000$1,000,000$ estimated ownership ( $N=1217$; approximately $3.31 \%$ of all non-VR games). Next, we employed Steam and SteamSpy API to gather additional information like game title, app ID, categories, user tags, release dates, ownership, and user ratings. This data allowed for coding of each game's Steam Store page.

\subsection{Content Analysis Coding Procedure}

Coding was performed through data scraping programs and human coders who can recognize program attributes scrapers miss. The data scrapers identified "tags," typically single-word descriptions of games including genre, associated with the programs. Three human coders adhered to categories derived from Lucas and Sherry [18] because they were developed from previous research and industry standards [18:511]; provided a middle ground between unmanageably large/intricate and small/broad counterparts; and provided clear genre descriptions. After initial review of the corpus, we modified the list by adding present genres from other classification systems, such as music/rhythm. The following genres resulted: Action, Adventure, Card/Dice, Classic Board Games, Fantasy/RPG, Fighter, Flight, Music/Rhythm, Puzzle, Quiz/Trivia, Racing/Speed, Shooter, Simulation, Sports, Strategy, and Turn-Based Strategy. We chose to rely on human coders because they are better able to categorize games systematically and consistently. We plan to compare this approach to player-generated tags in future work.

Three independent coders were trained on the coding protocols for identifying content (e.g., Action: a game that relies on a player's reflexes, coordination, or reaction time). Multiple genres could be assigned to each experience. A minimal acceptable threshold of $\alpha=.7$ was established, which all variables met or exceeded. Cohen's Kappa was not used due to the highly skewed nature of the data. 


\section{Preliminary Findings}

\subsection{What traditional game genres are most common in Steam-provided VR experiences?}

The five most commonly featured genres in VR were Action (45\%), Shooter (30\%), Simulation (22\%), Adventure (13\%), and Puzzle (12\%). The median number of genres was 1 for VR-only experiences (in contrast to 4 for non-VR games).

\subsection{Are user ratings and downloads higher for certain traditional genres in VR experiences?}

We created a "ratings" variable by dividing the number of positive (thumbs up) ratings by the total number of days the game had been published to control for the difference in the time a game was available to play and rate.

A stepwise linear regression was calculated to examine the connection between VR genres and the ratings variable. A significant regression equation was found $(F(2,136)=15.57, p<.001)$ such that experiences with Action $(t=2.37, \mathrm{p}=.02)$ and Music/Rhythm $(t=4.78, p<.001)$ genres were more likely to have positive ratings than other genres. Since they did not meet required assumptions for linear regressions, Classic Board Games, Card/Dice, Fantasy/RPG, Quiz/Trivia, Racing/Speed, Sports, Strategy, and Turn-Based Strategy could not be statistically analyzed in the VR condition. Linear regression requires the number of cases in a condition to be greater than the total number of conditions being regressed (16, in this analysis).

\subsection{Do VR and non-VR experiences differ with respect to traditional game genre availability and user ratings?}

4.3.1 Genre Availability. A series of chi-square tests of independence were performed to examine the relative availability of different genres in VR and non-VR experiences. Each test examined an individual genre to see if it existed equally within both modalities, or if one had a higher representation of that genre.

The genres that occur less frequently in VR than in non-VR experiences are Action $\left(\chi^{\wedge} 2(2, \quad n=1336)=60.603, \quad p<.001\right)$; Adventure $\left(\chi^{\wedge} 2(2, \quad n=1336)=178.178, \quad p<.001\right)$; Fighter $\left(\chi^{\wedge} 2(2\right.$, $n=1336)=67.488, p<.001)$; Shooter $\left(\chi^{\wedge} 2(2, n=1336)=42.870, p<.001\right)$; and Fantasy/RPG $\left(\chi^{\wedge} 2(2, \mathrm{~N}=1336)=81.815, p<.001\right)$.

The genres that occur more frequently in VR than in non-VR experiences are Simulation $\left(\chi^{\wedge} 2(2, n=1336)=15.070, p<.001\right)$ and Music/Rhythm $\left(\chi^{\wedge} 2(2, n=1336)=9.467, p=.002\right)$. Classic Board Games $\left(\chi^{\wedge} 2(2, \quad n=1336)=2.262, \quad p=.133\right)$ and Puzzle $\left(\chi^{\wedge} 2(2\right.$, $n=1336)=3.802, p=.051)$ appear to occur more frequently in VR, but the difference is not statistically significant. Chi-square tests could not be run on Dice, Flight, Quiz/Trivia, Racing/Speed, Sports,
Strategy, and Turn-Based Strategy due to too few cases from these genres in our VR sample (which suggests they occur more frequently in non-VR content).

4.3.2 User Ratings. A linear regression was performed to examine differences in ratings between VR and non-VR experiences using the same ratings metric from $R Q_{2}$. There was a significant effect $(t(1334)=-03.13, p=.002)$, such that non-VR games $(M=22.56$, $S D=76.85)$ attained more positive ratings than VR experiences $(M=2.16, S D=9.02)$.

Within non-VR games, a significant regression equation of genre on user rating was found $(F(3,1993)=8,09, p<.001)$. Strategy $(t=3.21, p=.001)$, Sports $(t=2.82, p<.005)$; and Shooter $(t=2.35$, $p=.019)$ games are more likely to have positive ratings. These results contrast with earlier findings that within VR experiences, Action and Music/Rhythm are most likely to receive positive ratings.

\section{Discussion}

In addition to charting the current VR landscape for researchers, our preliminary findings uncovered surprising insights for industry developers and marketers.

Players more positively review certain genres in VR (i.e., Action and Music/Rhythm). This makes intuitive sense. Given that a primary feature of VR is its depth of immersion, Action games where players find themselves in intense situations and environments would be entertaining. Furthermore, movement is a contributing factor to such immersion, upon which Music/Rhythm games capitalize.

By contrast, genres like Strategy and Sports are relatively rare in VR. While a Music/Rhythm game makes the most of player movements, VR hardware constrains the aggressive, complicated actions associated with Sports games. These genres also have existing conventions in terms of player control and interface that are difficult to translate. A player who could, on a console, press a button to perform a bicycle kick would find the same maneuver difficult (and dangerous) to execute while in an HMD.

We also found that some genres that require long periods of gameplay (e.g., Adventure, Shooter) are less popular in VR than non-VR. Player preferences for shorter experiences could be linked to hardware discomfort, including heat, eye strain, physical fatigue, and simulator sickness. While Shooter games are immersive given their first-person perspective, play in contemporary HMDs may still cause motion sickness. This pattern could also relate to being "disconnected" from the natural environment for long periods of time, unlike a traditional video game. While promising, each possibility calls for follow-up studies.

Finally, although VR experiences were more harshly reviewed than non-VR in the data set, this may be because of the medium's relative infancy: fewer available experiences are more frequently 
downloaded, but may be less sophisticated than non-VR games; so they are more harshly reviewed. This may also reflect a willingness to experiment with purchasing. Unlike video games, consumer VR preferences may still be forming.

In total, our preliminary results suggest VR users tend to favor shorter experiences that maximize physical involvement (within reason). Knowing what styles attract and are enjoyed by players can inform developers' creative choices.

\subsection{Future Directions}

This research is a first step in defining the role genre plays in the consumption of VR content. As analyses of popular commercial VR styles are rare, our findings provide valuable measurements by which developers can assess what genres do well in the marketplace. However, this work in progress is still in its early stages: We did not include analysis of "VR Supported" products, which contain both VR and non-VR content, in part because differentiated rating information is difficult to ascertain. We plan to expand our analysis into this category, which could impact creators who wonder if their games would find success in HMDs, as was the case with No Man's Sky, which was successfully ported to VR after years of trepidation by the publisher [19].

Further, we acknowledge that traditional genres offer a limited perceptual filter to articulate how VR experiences differ. We will combine these findings with other factors, including presence, perspective-taking, and interactivity, in order to rethink and develop a more comprehensive classification model. Technical factors, including controls and hardware, also impact genre preference: a Shooter played in mobile VR is a vastly different experience compared to the latest commercial HMD and controllers. Each element informs designer and player choices, along with the popularity of certain styles.

\subsection{Challenges}

Inconsistent standards challenged this investigation, which is still highly hypothetical. Genre systems' lack of uniformity impacts any similar study's methodology because different classifications might yield other results in regard to popularity, although our findings were statistically reliable. This shortcoming also exists at the platform level: Steam treats every application as a game, including utilities and film. We coded based on traditional game genres, but realize that more expansive classifications are required to account for experiences that do not conform to game categories. Especially given the fluid interpretation of genre among researchers and consumers alike, a mixed method and qualitative approach, such as thematic analysis of user comments, as well as comparing human coding to the data scraped tags, seems necessary to advance this initial work. Finally, the SteamSpy API only recorded large ranges for download rates, which hinders precise analysis. However, VR and digital game makers do not regularly publish these figures. Thus, SteamSpy offers one of the better avenues for gaining this information [28].

\section{Conclusions}

While affiliated with games and gaming, VR is maturing into a medium with unconventional features and styles as well as novel approaches to familiar genres. This work analyzed how players engage with the medium by first reviewing and testing the relationship between genre, games, and VR based on the most popular genres currently available to users in Steam. We found Action, Shooter, and Simulation experiences to be most common; Action and Music/Rhythm to be the most highly rated; and Simulation and Music/Rhythm to occur at a statistically higher rate in VR compared to non-VR; finally, we discovered that VR experiences are less likely to receive positive ratings compared to $2 \mathrm{D}$ video games. Ultimately the study serves as an important start in discerning the types of VR content users gravitate toward, which can benefit designers, industry, and players alike in future production.

The work underscores the value of utilizing genre in the appraisal of emerging media. Yet, when mapping how different styles manifest in VR, we see the need for a more comprehensive assessment of genre that moves beyond content and considers psychological and technical attributes and effects. After all, many users may be using genre to help select their very first commercial VR experiences, and it is important that these categories actually reflect the reality of content they consume.

\section{ACKNOWLEDGMENTS}

We would like to thank our research assistants: Ann Desrochers, Matthew Klein, and Lindsey Murrell.

\section{REFERENCES}

[1] Jeremy Bailenson. 2018. Experience on Demand: What Virtual Reality Is, How It Works, and What It Can Do. (1st. ed.) W. W. Norton \& Company. New York, NY.

[2] Jeremy N. Bailenson, Nick Yee, Jim Blascovich, Andrew C. Beall, Nicole Lundblad, and Michael Jin. 2008. The Use of Immersive Virtual Reality in the Learning Sciences: Digital Transformations of Teachers, Students, and Social Context. Journal of the Learning Sciences 17, 1, 102-141.

[3] Frank Biocca. 1996. Chapter 3 Intelligence augmentation: The vision inside virtual reality. Cognitive Technology - In Search of a Humane Interface, 59-75. https://doi.org/10.1016/s0166-4115(96)80023-9

[4] Jim Blascovich and Jeremy Bailenson. 2011. Infinite Reality: The Hidden Blueprint of Our Virtual Lives. William Morrow Paperbacks (1st. ed.). New York, NY.

[5] Pawel Dobrowolski, Krzysztof Hanusz, Bartosz Sobczyk, Maciek Skorko, and Andrzej Wiatrow. 2015. Cognitive enhancement in video game players: The role of video game genre. Computers in Human Behavior 44, 59-63.

[6] Luther Elliott, Andrew Golub, Geoffrey Ream, and Eloise Dunlap. 2012. Video game genre as a predictor of problem use. Cyberpsychology, Behavior and Social Networking 15, 3, 155-161.

[7] Ali Faisal and Mirva Peltoniemi. 2018. Establishing Video Game Genres Using Data-Driven Modeling and Product Databases. Games and Culture 13, 1, 20-43.

[8] Maxwell Foxman. 2019. United We Stand: Platforms, Tools and Innovation With the Unity Game Engine. Social Media + Society 5, 4 . https://doi.org/10.1177/2056305119880177

[9] Julian Frommel, Kim Fahlbusch, Julia Brich, and Michael Weber. 2017. The Effects of Context-Sensitive Tutorials in Virtual Reality Games. In Proceedings of the Annual Symposium on Computer-Human Interaction in Play, (CHI PLAY '17), October 15-17, Amsterdam, NL, 367-375.

[10] M. P. Jacob Habgood, David Wilson, David Moore, and Sergio Alapont. 2017. HCI Lessons From PlayStation VR. In Extended Abstracts Publication of the Annual Symposium on Computer-Human Interaction in Play (CHI PLAY '17 Extended Abstracts), October 15-17, Amsterdam, NL, 125-135.

[11] Carrie Heeter. 1992. Being There: The Subjective Experience of Presence. Presence: Teleoperators and Virtual Environments 1, 2, 262-271.

[12] Stephanie Heintz and Effie Lai-Chong Law. 2015. The Game Genre Map: A Revised Game Classification. In Proceedings of the 2015 Annual Symposium on 
Computer-Human Interaction in Play (CHI PLAY '15), October 5-7, London, United Kingdom, 175-184.

[13] Daniel Johnson, Peta Wyeth, Penny Sweetser, and John Gardner. 2012 Personality, Genre and Videogame Play Experience. In Proceedings of the 4th International Conference on Fun and Games (FnG '12), September 4-6, Toulouse, France, 117-120.

[14] Marcel Jonas, Steven Said, Daniel Yu, Chris Aiello, Nicholas Furlo, and Douglas Zytko. 2019. Towards a Taxonomy of Social VR Application Design. In Extended Abstracts of the Annual Symposium on Computer-Human Interaction in Play Companion Extended Abstracts (CHI PLAY '19 Extended Abstracts), October 2225, Barcelona, Spain, 437-444.

[15] Martijn J. L. Kors, Erik D. van der Spek, Gabriele Ferri, and Ben A. M. Schouten. 2018. You; the Observer, Partaker or Victim. Delineating Three Perspectives to Empathic Engagement in Persuasive Games Using Immersive Technologies. In Proceedings of the 2018 Annual Symposium on Computer-Human Interaction in Play Companion Extended Abstracts (CHI PLAY '18 Extended Abstracts), October 28-31, Melbourne, Australia, 493-501.

[16] Andrey Krekhov, Sebastian Cmentowski, and Jens Krüger. 2018. VR Animals: Surreal Body Ownership in Virtual Reality Games. In Proceedings of the 2018 Annual Symposium on Computer-Human Interaction in Play Companion Extended Abstracts (CHI PLAY '18 Extended Abstracts), October 28-31, Melbourne, Australia, 503-511.

[17] Matthew Lakier, Lennart E. Nacke, Takeo Igarashi, and Daniel Vogel. 2019. Cross-Car, Multiplayer Games for Semi-Autonomous Driving. In Proceedings of the Annual Symposium on Computer-Human Interaction in Play (CHI PLAY '19), October 22-25, Barcelona, Spain, 467-480.

[18] Kristen Lucas and John L. Sherry. 2004. Sex Differences in Video Game Play: A Communication-Based Explanation. Communication Research 31, 5, 499-523.

[19] Mike Minotti. 2019. No Man's Sky is getting VR support. VentureBeat. Retrieved July 14, 2020 from https://venturebeat.com/2019/03/25/no-mans-sky-is-gettingvr-support-on-ps4-and-pc/

[20] David B. Nieborg and Thomas Poell. 2018. The Platformization of Cultural Production: Theorizing the Contingent Cultural Commodity. New Media \& Society 20, 11, 4275-4292.

[21] Federica Pallavicini and Alessandro Pepe. 2019. Comparing Player Experience in Video Games Played in Virtual Reality or on Desktop Displays: Immersion, Flow, and Positive Emotions. In Extended Abstracts of the Annual Symposium on Computer-Human Interaction in Play Companion Extended Abstracts (CHI PLAY '19 Extended Abstracts), October 22-25, Barcelona, Spain, 195-210.
[22] Nicole Peever, Daniel Johnson, and John Gardner. 2012. Personality \& video game genre preferences. In Proceedings of The 8th Australasian Conference on Interactive Entertainment: Playing the System (IE '12), Auckland, NZ, July 21-22, $1-3$.

[23] John Porter III, Matthew Boyer, and Andrew Robb. 2018. Guidelines on Successfully Porting Non-Immersive Games to Virtual Reality: A Case Study in Minecraft. In Proceedings of the 2018 Annual Symposium on Computer-Human Interaction in Play (CHI PLAY '18), October 28-31, Melbourne, Australia, 405415.

[24] Ute Ritterfeld and René Weber. 2006. Video Games for Entertainment and Education. Playing video games: Motives, responses, and consequences, 399-413.

[25] Chek Tien Tan, Tuck Wah Leong, Songjia Shen, Christopher Dubravs, and Chen Si. 2015. Exploring Gameplay Experiences on the Oculus Rift. In Proceedings of the 2015 Annual Symposium on Computer-Human Interaction in Play (CHI PLAY '15), October 5-7, London, United Kingdom, 253-263.

[26] Gustavo F. Tondello and Lennart E. Nacke. 2019. Player Characteristics and Video Game Preferences. In Proceedings of the Annual Symposium on ComputerHuman Interaction in Play (CHI PLAY '19), October 22-25, Barcelona, Spain, 365-378.

[27] Gustavo F. Tondello, Rina R. Wehbe, Rita Orji, Giovanni Ribeiro, and Lennart E. Nacke. 2017. A Framework and Taxonomy of Videogame Playing Preferences. Proceedings of the Annual Symposium on Computer-Human Interaction in Play (CHI PLAY '17). Amsterdam, NL, October 15-18, 329-340 https://doi.org/10.1145/3116595.3116629

[28] Joost van Dreunen. 2018. Welcome to the New Data Reality in Video Games. SuperData, a Nielsen Company. Retrieved July 13, 2020 from https:/www.superdataresearch.com/blog/welcome-to-the-new-data-realityin-video-games

[29] Lotte Vermeulen and Jan Van Looy. 2016. "I Play So I Am?" A Gender Study into Stereotype Perception and Genre Choice of Digital Game Players. Fournal of Broadcasting \& Electronic Media 60, 286-304. https://doi.org/10.1080/08838151.2016.1164169

[30] Zhan Ye. 2004. Genres as a Tool for Understanding and Analyzing User Experience in Games. In CHI '04 Extended Abstracts on Human Factors in Computing Systems (CHI EA '04), Vienna, Austria, April 24-29, 773-774. 